\title{
Intraductal Ultrasound for High-Risk Patients: When Will the Last Be First?
}

\author{
Pietro Fusaroli · Giancarlo Caletti
}

Published online: 4 June 2014

(c) Springer Science+Business Media New York 2014

Endoscopic ultrasound (EUS) has excellent overall sensitivity $(94 \%)$ and specificity $(95 \%)$ for the diagnosis of choledocholithiasis [1]. EUS is also very accurate in the diagnosis of common bile duct (CBD) sludge, with a sensitivity up to $90 \%$ and specificity of $97 \%$ (personal data). By performing EUS first in patients with suspected CBD stones, endoscopic retrograde cholangiopancreatography (ERCP) with endoscopic sphincterotomy (EST) may be safely avoided in two-thirds of the cases. On the other hand, ERCP with EST is appropriate when the presence of CBD stones has been documented by EUS. As a result, a EUS-based selection of patients for therapeutic ERCP significantly reduces complication rates [2, 3].

The American Society of Gastrointestinal Endoscopy issued a risk-stratified algorithm for patients with suspected CBD stones, EUS being indicated first in those with intermediate probability of CBD stones [4]. Nevertheless, ERCP is usually recommended as the first step in those with a high probability of CBD stones. Yet, this approach is not always followed for two reasons: first, in order to minimize the risk of medico-legal claims, many endoscopists want to be on the safe side and proceed to ERCP only after CBD stones have been clearly demonstrated; second, in elderly and fragile patients, stent positioning in the CBD without EST may represent a safer alternative to EST plus stone retrieval, in order to minimize procedure-related

P. Fusaroli $(\bowtie) \cdot$ G. Caletti

GI Unit, Department of Medical and Surgical Sciences,

University of Bologna/Hospital of Imola, Via Montericco 4, 40026 Imola, BO, Italy

e-mail: pietro.fusaroli@unibo.it risks. In both instances, it is clear that EUS or, alternatively, magnetic resonance cholangiopancreatography (MRCP) is required to plan optimal therapeutic strategy.

In this issue of Digestive Diseases and Sciences, a Korean study authored by Kim et al. [5] reported about the usefulness of intraductal ultrasound (IDUS) as an alternative to EUS in high-risk patients undergoing ERCP. Patients were referred to ERCP based on the presence of jaundice (serum bilirubin $\geq 3 \mathrm{mg} / \mathrm{dL}$ ) and clinical ascending cholangitis or dilated CBD as assessed with ultrasonography, computed tomography, or MRCP. In all cases, no CBD stones or other causes of CBD obstruction were identified prior to ERCP. Thus, it appears that patients were scheduled for ERCP based only on high-risk clinical presentation without prior EUS.

Ninety-five patients were prospectively studied with IDUS if ERCP failed to identify filling defects inside the CBD. IDUS identified stones or sludge in $58 \%(55 / 95)$ of the patients; $33 \%$ had stones (mean diameter $2.9 \mathrm{~mm}$, range $1-7 \mathrm{~mm}$ ); and $25 \%$ had sludge alone. IDUS findings were confirmed by extraction of stones in 31/31 patients and of sludge in 21/24 patients (97\% overall accuracy). MRCP of an unknown number of patients and ERCP had failed to identify small stones and sludge in the 55 patients with IDUS-identified stones or sludge.

Although there is no statistically significant difference between EUS and MRCP accuracy for the detection of choledocholithiasis [6], the sensitivity of MRCP is decreased in the setting of small $(<6 \mathrm{~mm})$ CBD stones, whereas EUS remains highly sensitive even for small stones. Similarly, it has been reported that EUS is more sensitive than ERCP in detecting stones smaller than $4 \mathrm{~mm}$ [7]. Based on the data reported in the Korean study, we can assume that accuracy of EUS and IDUS is somewhat equivalent for the diagnosis of CBD stones and sludge. 
Intraductal ultrasound is a relatively straightforward procedure, which is performed with high-frequency catheter probes (range 12-30 MHz). Two designs are available: thin rail-guided miniprobes, which can be introduced into the CBD over a guidewire, and standard miniprobes, which can be introduced "free-hand" into the CBD. Advantages of IDUS include very high diagnostic accuracy even for small stones, short procedure time, lack of radiation, and availability at any time during ERCP even without prior EST. Drawbacks of IDUS include limited depth of ultrasound view compared with EUS, which makes it impossible to fully investigate the pancreas and surrounding structures when other causes of CBD obstruction are to be ruled out, e.g., pancreatic cancer. Additionally, miniprobes work by mechanical scanning, which excludes the use of Doppler-based methods. Moreover, miniprobes can be used only in conjunction with few proprietary echo-processors by Olympus and Fujinon but not with all the ultrasound processing units, thereby limiting general availability of IDUS among endoscopic centers.

Miniprobes may constitute the only available tool for exploring the CBD in certain patients such as following gastric resection (Billroth II and Roux-en-Y interventions). In these patients, it is always very difficult or impossible to reach the ampulla of Vater with dedicated echoendoscopes, which in general are large and poorly maneuverable. Instead, the ampulla of Vater may be reached with a standard upper endoscope from where miniprobe assessment of the CBD can be performed. Alternatively to the intraductal scanning, accurate investigation of the CBD can also be achieved by transduodenal miniprobe scanning. In one study, the diagnostic accuracy of catheter-probe extraductal ultrasonography was comparable to that of conventional EUS for the detection of CBD stones [8].

Kim et al. [5] reported use of IDUS as a salvage technique to be performed at the time of ERCP. When patients are submitted directly to ERCP, based on high-risk clinical criteria without previous EUS or MRCP, if cholangiography fails to show any intraductal filling defect, it is less likely to proceed to EST for fear of unnecessary treatment. In the discussed study, therapeutic management was positively influenced by IDUS in $55 \%$ of the patients in whom EST would otherwise not have been performed because of negative cholangiography.

Interesting results with IDUS were reported in previous studies from other countries and from Korea as well, where experience with this technique appears to have been developed widely and thoroughly. Moon et al. [9] compared several techniques in the diagnosis of CBD stones in patients with suspected biliary pancreatitis. While dismal results for transabdominal ultrasound and computed tomography where reported, MRCP, ERCP, and IDUS showed sensitivity of 80,90 , and $95 \%$, respectively.
Similarly to Kim et al. [5] dilatation of the CBD was identified as a risk factor for decreased sensitivity for detecting choledocholithiasis by techniques other than IDUS. Another study reported that approximately half of the patients with echogenic CBD material visible on IDUS actually had biliary crystals on bile microscopy [10].

What are the main practical implications of the present Korean study? First, in the era of same-day EUS-ERCP for the diagnosis and treatment of CBD stones, IDUS appears to be a quick and accurate alternative. Secondly, compared to cholangioscopy, IDUS is much safer and less expensive. In fact, miniprobes are reusable and can last up to 100 examinations when properly handled. Nonetheless, proceeding directly to IDUS + ERCP without previous EUS or MRCP is warranted only in high-risk patients with a high probability of harboring CBD stones. In all other instances, including cases where malignancy has not been excluded, a more complete examination with EUS is warranted. Unlike IDUS and MRCP, dedicated EUS is the only technique that can provide high-resolution imaging of the whole pancreatobiliary structures and fine-needle aspiration when tissue confirmation is needed.

\section{References}

1. Tse F, Liu L, Barkun AN, Armstrong D, Moayyedi P. EUS: a meta-analysis of test performance in suspected choledocholithiasis. Gastrointest Endosc. 2008;67:235-244.

2. Polkowski M, Regula J, Tilszer A, Butruk E. Endoscopic ultrasound versus endoscopic retrograde cholangiography for patients with intermediate probability of bile duct stones: a randomized trial comparing two management strategies. Endoscopy. 2007;39: 296-303.

3. Karakan T, Cindoruk M, Alagozlu H, Ergun M, Dumlu S, Unal S. EUS versus endoscopic retrograde cholangiography for patients with intermediate probability of bile duct stones: a prospective randomized trial. Gastrointest Endosc. 2009;69:244-252.

4. ASGE Standards of Practice Committee, Maple JT, Ben-Menachem T, Anderson MA, et al. The role of endoscopy in the evaluation of suspected choledocholithiasis. Gastrointest Endosc. 2010;71:1-9.

5. Kim DC, Moon JH, Choi HJ et al. Usefulness of Intraductal Ultrasonography in Icteric Patients with Highly Suspected Choledocholithiasis Showing Normal Endoscopic Retrograde Cholangiopancreatography. Dig Dis Sci. (Epub ahead of print). doi:10. 1007/s10620-014-3127-1.

6. Verma D, Kapadia A, Eisen GM, Adler DG. EUS vs MRCP for detection of choledocholithiasis. Gastrointest Endosc. 2006;64: 248-254.

7. Petrov MS, Savides TJ. Systematic review of endoscopic ultrasonography versus endoscopic retrograde cholangiopancreatography for suspected choledocholithiasis. Br J Surg. 2009;96: 967-974.

8. Wehrmann T, Martchenko K, Riphaus A. Catheter probe extraductal ultrasonography vs. conventional endoscopic ultrasonography for detection of bile duct stones. Endoscopy. 2009;41: 133-137. 
9. Moon JH, Cho YD, Cha SW, et al. The detection of bile duct stones in suspected biliary pancreatitis: comparison of MRCP, ERCP, and intraductal US. Am J Gastroenterol. 2005;100: 1051-1057.
10. Kim BJ, Kang P, Lee JK, et al. Are the echogenicities on intraductal ultrasonography really biliary microlithiasis? Dig Dis Sci. 2010;55:836-841. 